\title{
Identificación de riesgos naturales y antrópicos en el casco urbano del municipio de Quibdó: estudio de caso
}

\section{Identification of the natural and antropics hazards in the urban area of Quibdó: case study}

\author{
Yenecith TorRes Allín*
}

\section{RESUMEN}

Los riesgos naturales y antrópicos de mayor repercusión en el casco urbano del municipio de Quibdó se determinaron mediante el diligenciamiento de encuestas a la comunidad y compilación de registros históricos de desastres ocurridos en el municipio. Un total de 856 encuestas diligenciadas, muestran a las inundaciones y vientos fuertes representados en $27 \%$ y 25\% respectivamente, como las amenazas de mayor ocurrencia. Los registros históricos relacionados con el número de viviendas averiadas en los últimos diez años, revelan a los vientos fuertes con 144 viviendas afectadas como los eventos de mayor significancia (40\%). Al considerar 123 registros de desastres ocurridos en la ciudad de Quibdó en los últimos 80 años, las inundaciones (43\%) y los deslizamientos (25\%), son las amenazas más representativas. En adición, las inundaciones son los eventos que más pérdidas económicas le han emanado al municipio de Quibdó a través de la historia.

Palabras clave: Riesgos; Vulnerabilidades; Amenazas; Encuestas; Registros históricos.

\begin{abstract}
Natural and anthropogenic hazards in the urban area of Quibdo, were determined by the diligence of the community surveys and compilation of historical records of disasters in the city. The 856 processed surveys show that floods and strong winds are the most occurrence threats represented by $27 \%$ and $25 \%$ respectively. Historical records relating to the number of damaged houses in the last ten years, show strong winds with 144 houses affected, as the events of major significance, represented by 40\%. Take into consideration 123 disasters records to the city in the last 80 years, flooding and landslides with $43 \%$ to $25 \%$ are the most representative hazards. In addition, floods are the events that most economic losses have cost to Quibdo city in the history.
\end{abstract}

Keywords: Hazards; Vulnerability; Threat; Surveys; Historical records.
* Investigadora de Proyectos Especiales, Instituto de Investigaciones Ambientales del Pacífico (IIAP)

e-mail:yene8025@yahoo.com

Recibido: agosto 14, 2009

Aceptado: septiembre 3, 2009

\section{INTRODUCCIÓN}

El riesgo es definido como la probabilidad de que se presenten pérdidas o consecuencias económicas y sociales debido a la ocurrencia de un fenómeno peligroso. Se obtiene de relacionar la amenaza o probabilidad de ocurrencia de un evento de cierta intensidad, con la vulnerabilidad o potencia- lidad que tienen los elementos expuestos a ser afectados por el evento (Lavell, 1996).

Cada año ocurren en el mundo desastres que ocasionan graves pérdidas económicas y humanas. Sólo para el período 1970-1989 los daños económicos fueron estimados en 50.000 millones de dólares y se registraron más de 1,6 millones de víctimas, considerando los episodios con más de 20 mil muertes (Ayala-Carcedo, 1993). En los últimos 50 años, los 
desastres naturales han provocado la pérdida de más de 2,8 millones de vidas humanas en el planeta, incrementándose en 6\% cada año desde 1960 el número de personas afectadas, el doble de la tasa de crecimiento demográfico mundial(DIRDN, 1996).

En Colombia se han visto afectadas más de cuatro millones de personas por desastres naturales en el período comprendido entre 1993 y 2000, la mayoría por inundaciones y sismos (Conpes 3146, 2001). Para el departamento del Chocó en los últimos diez años se han informado más de 100,600 personas afectadas por inundaciones (DNPAD, 2007).

De todas las amenazas que pueden presentarse en el municipio de Quibdó, son tal vez las inundaciones y deslizamientos las más evidentes, las cuales se presentan sobre todo en sectores ampliamente habitados de los barrios Kennedy, los Álamos, Porvenir, Chambacú entre otros (POT, 2004). Esta situación que se debe principalmente a precipitaciones anuales que sobrepasan los 7,400 mm (IGAC INGEOMINAS, 2006) y a que las tendencias de crecimiento de la ciudad se han llevado a cabo de forma no planificada, creando una configuración urbana improvisada y carente de orden. En adición, la vulnerabilidad aumenta debido a la cultura del agua caracterizada por la presencia de núcleos poblados organizados de forma lineal a orillas de las riveras de los ríos y al fenómeno de desplazamiento que de acuerdo con los informes de la ACNUR (2006) asciende a más de 28.826 personas afectadas, con lo cual ha aumentado la construcción en zonas altamente amenazadas como son las laderas y orillas de los cuerpos de agua, disminuyendo a su vez los procesos de saneamiento del municipio, tales como generación de desechos sólidos y vertimiento directo de aguas servidas a los ríos (Conpes 3470, 2007). En adición, el estado de la red de alcantarillado en todo el municipio es crítico, en muchos sectores ha colapsado y/o colmatado con el agravante de existir drenajes superficiales (DASALUD, 2007).

Esta problemática sumada a la falta de estudios sistemáticos sobre pérdidas económicas y ambientales asociadas con eventos de alta recurrencia, como inundaciones y deslizamientos localizados; con que el conocimiento de las vulnerabilidades físicas, sociales, económicas y culturales del municipio ante estas amenazas es incipiente y existe deficiencia en la incorporación del tema de planificación en el esquema de ordenamiento territorial, obliga a identificar la ocurrencia e intensidad de fenómenos naturales y antrópicos que vienen provocando daños a la comunidad.

\section{MATERIALES Y MÉTODOS}

Área de estudio. Quibdó es la capital del departamento del Chocó situado en el occidente de Colombia. La ciudad se encuentra a orillas del río Atrato, uno de los principales afluentes del país y una de las zonas con más alta pluviosidad del mundo. Se localiza a los $05^{\circ} 41^{\prime} 41^{\prime \prime}$ de latitud norte y $76^{\circ}$ $39^{\prime} 40^{\prime \prime}$ de longitud oeste. Posee una altura sobre el nivel del mar de $43 \mathrm{~m}$ y temperatura media de $28^{\circ} \mathrm{C}$. Dista $869 \mathrm{~km}$ de la capital de la República. El área municipal tiene $6.032 \mathrm{~km}^{2} \mathrm{y}$ limita por el norte con Bojayá y el departamento de Antioquia, por el este con el departamento de Antioquia y los municipios del Carmen y Lloró, por el sur con Lloró, Tadó, Cantón de San Pablo y Alto Baudó, y por el oeste con Alto Baudó, y Bojayá.

El rasgo geográfico más importante del municipio lo constituye la Serranía del Baudó, la cual consiste en una serie de colinas alineadas de dirección N-S, con alturas que alcanzan hasta $500 \mathrm{msnm}$. Este relieve colinado forma vertientes escarpadas. Otro rasgo notable lo constituye la llanura de inundación del río Atrato, que alcanza hasta $10 \mathrm{~km}$ de amplitud, formando una extensa zona de pantanos, la cual permanece inundada durante casi todo el año. El río Atrato y sus tributarios mayores conforman las vías de comunicación de la zona; la cantidad excedente de agua en toda la zona ha formado una red de drenaje muy densa a lo largo de todos los cursos de agua (IGAC-INGEOMINAS, 2006).

Cuenta con una población de 112,866 habitantes, de los cuales 101,134 habitan en el área urbana (Dane, 2005).

Métodos. Basados en reuniones realizadas con el Comité de Atención y Prevención de Desastres Municipal y entrevistas personalizadas con conocedores del tema, se elaboraron encuestas dirigidas a la comunidad en general que permitieran obtener un listado de las principales amenazas y vulnerabilidades del casco urbano del municipio de Quibdó.

Considerando un total de 25,000 viviendas para el casco urbano del municipio (DANE 2005), se calculó el número de viviendas por comunas sobre las cuales se aplicarían las listas de chequeo. Se realizaron 856 encuestas de manera aleatoria en diferentes sectores de las seis comunas que hacen parte del casco urbano del municipio. Una vez diligenciadas las listas de chequeo, la información obtenida fue depurada y analizada con ayuda de la herramienta SPSS versión 15.0

Posteriormente, en la página del Sistema de Inventario de Efectos de Desastres en la Comunidad Andina (http:// online.desinventar.org CORPOSSO-La Red, 2007) se colectó información de los eventos naturales y antrópicos ocurridos en el casco urbano del municipio de Quibdó, en el período comprendido entre 1935 hasta 2007; los eventos ocurridos en 2008 se tomaron de la página del Sistema Nacional para la Atención y Prevención de Desastres (www.sigpad.gov.co); los datos fueron depurados de acuerdo con las necesidades del presente estudio y analizados con la herramienta estadística SPSS versión 15.0.

Finalmente, los datos obtenidos en campo con los facilitados por diferentes entidades del nivel nacional, fueron comparados y analizados con el objeto de identificar los principales riesgos de carácter natural y antrópico del casco urbano del municipio de Quibdó. 


\title{
Bioetnia Volumen 5 No 2 (julio-diciembre), 2008
}

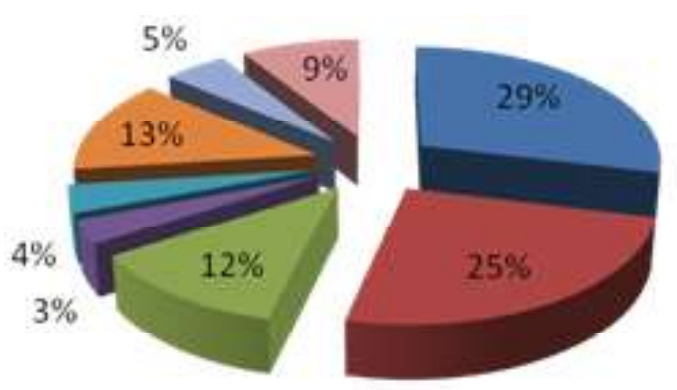

$=$ Inundaciones
$=$ Vientos fuertes
$=$ Deslizamientos
$=$ Sismos
$=$ Incendios
$=$ Inundaciones, vientos fuertes
= Inundaciones, deslizamientos

\section{Gráfica 1. Amenazas naturales y antrópicas del casco urbano del municipio de Quibdó de acuerdo con las encuestas realizadas}

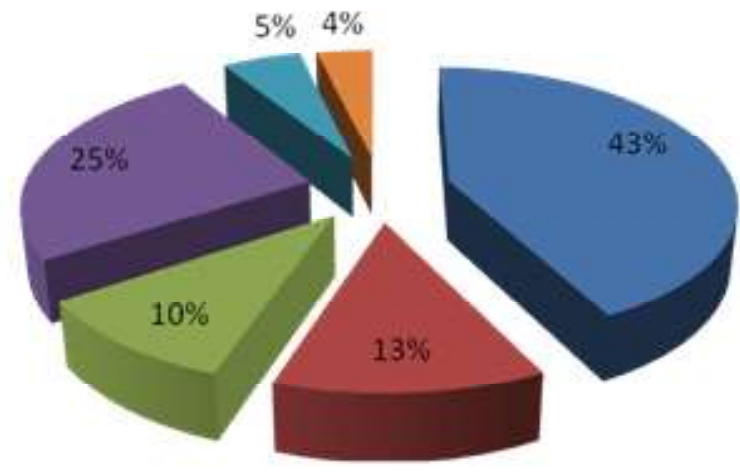

\author{
- Inundaciones \\ - Incendios \\ Vientos fuertes \\ Deslizamientos \\ Sismos \\ Otros
}

\section{Gráfica 2. Amenazas presentes en el casco urbano del municipio de Quibdó de acuerdo con los registros históricos 1935-2008}

\section{RESULTADOSYDISCUSIÓN}

De acuerdo con las entrevistas realizadas a la comunidad del casco urbano de la ciudad de Quibdó, se han presentado en los últimos años inundaciones, vientos fuertes y deslizamientos principalmente. Las amenazas más destacadas son las inundaciones y vientos fuertes representados en $29 \%$ y $25 \%$ respectivamente. En algunos sectores ocurren varias amenazas a la vez, como es el caso de las inundaciones y vientos fuertes, y vientos fuertes y deslizamientos representados en $13 \%$ y $9 \%$ respectivamente (Gráfica 1 ).

Considerando 125 registros de eventos reportados por el Sistema de Inventario de Efectos de los Desastres para el municipio de Quibdó (1935-2008), las inundaciones con 43\% y deslizamientos con $25 \%$, son las amenazas más representativas, seguidas de los incendios $(13 \%)$ y vientos fuertes (vendavales) (10\%) (Gráfica 2).

Sin embargo, si se consideran los niveles de afectación de dichos eventos por las 364 viviendas que han sido averiadas durante los últimos 10 años, los vientos fuertes (40\%) constituyen los eventos de mayor significancia durante los 10 años de reporte, seguidos en su orden de las inundaciones
(34\%) y los deslizamientos (26\%). Lo anterior denota que la presencia de vientos fuertes constituye una amenaza de alta ocurrencia que ha venido afectado de manera sustancial a los habitantes del casco urbano del municipio de Quibdó.

El riesgo por inundación ha aumentado por la obstrucción y la sedimentación del río Atrato; $23 \%$ de los habitantes del municipio disponen los residuos en ríos o quebradas; como gran parte de las viviendas se encuentran ubicadas en las orillas de diferentes tributarios del río Atrato, para ellos «resulta más cómodo arrojar las basuras al río que esperar a que pase el carro recolector», aunque $60 \%$ de los encuestados manifiesta que la frecuencia de recolección es de dos y tres veces a la semana. De igual forma, $80 \%$ del total de las viviendas encuestadas no cuentan con servicio de alcantarillado, por lo cual existe alta proliferación de vectores, que representan una gran amenaza para la salud de los habitantes.

Según el mapa geológico de Colombia (AIS-INGEOMINAS, 1997), el Andén Pacífico Colombiano presenta riesgo sísmico alto; los eventos por sismos en el casco urbano del municipio de Quibdó de acuerdo con las encuestas realizadas se encuentran representados sólo en 3\%, y los reportes de sismos registrados para el municipio de Quibdó por el Sistema 
de Inventario de Efectos de los Desastres desde el año 1962 hasta 2007 representan5\%, lo cual puede deberse a que el municipio de Quibdó no ha sido epicentro de ninguno de los sismos ocurridos, y estos no se presentan desde 1995.

Aunque gran parte de las zonas del casco urbano del municipio de Quibdó, presentan una estabilidad relativa y firmeza del suelo, esta desaparece en determinadas áreas predominando en ellas el proceso de deslizamiento, derrumbes y erosiones. La alta ocurrencia de este fenómeno está dada por las altas precipitaciones del municipio y por la destrucción de los bosques o deforestación para la construcción de viviendas.

El municipio ha sufrido en su historia varios incendios masivos que han condicionado la evolución urbanística de la misma, información que es corroborada con los incendios reportados por el Sistema de Inventario de Efectos de los Desastres representados en un $17 \%$. Sin embargo, de acuerdo con las encuestas realizadas, para los habitantes del municipio de Quibdó esta amenaza pasa a tener poca importancia (4\%), porque gran parte de los incendios de gran relevancia ocurrieron en la década de 1970 en zonas específicas del municipio. En adición no se cuenta con las medidas preventivas necesarias porque existe una proporción de $23 \%$ en construcciones mixtas (madera y cemento) y $22 \%$ en madera, datos que sumados a la ubicación de redes eléctricas en zonas muy cercanas a las viviendas y a la falta de cuerpo de bomberos aumenta el riesgo por incendios.

En relación con las pérdidas económicas generadas por eventos naturales y/o antrópicos, $62 \%$ de los encuestados no ha sufrido pérdida por riesgos, mientras $33 \%$ manifiesta haberlas tenido, siendo las principales causas las inundaciones y deslizamientos. Para aquellos que han sufrido pérdidas, se estima en un valor de uno a tres millones de pesos, representados en $28 \%$ y $4 \%$ respectivamente. Frente a las amenazas potenciales, $50 \%$ de las familias están dispuestas a reubicarse; de este porcentaje $18 \%$ contribuiría para la misma, de los cuales $14 \%$ aportarían en mano de obra y $4 \%$ con dinero $\mathrm{y} / \mathrm{o}$ materiales.

Según el DNPAD, las inundaciones con \$646’075.614 (seiscientos cuarenta y seis millones setenta y cinco mil seiscientos catorce pesos) invertidos, son los eventos que más le han costado al municipio de Quibdó en los últimos 10 años, representados en $83 \%$ del total de las inversiones. Los recursos provienen del Fondo Nacional de Calamidades y han sido destinados principalmente para alimentación, menajes y materiales para construcción.

A pesar del alto riesgo ante la presentación de desastres en el municipio, solo $8 \%$ de los encuestados han recibido capacitación relacionada con esta temática y solo $6 \%$ de los hogares encuestados han participado en simulacros contra emergencias ambientales.

\section{AGRADECIMIENTOS}

La autora agradece al equipo contratado para el levantamiento de encuestas y apoyo en la formulación del proyecto. A la comunidad del casco urbano del municipio de Quibdó por su disposición en el desarrollo de encuestas y a las diferentes entidades que hacen parte del Comité de Atención y Prevención de Desastres Municipal por la información suministrada y apoyo incondicional.

\section{BIBLIOGRAFÍA}

Alcaldía Municipal de Quibdó. 2004. Plan de Ordenamiento Territorial Municipio de Quibdó. Diagnóstico Territorial.

Alto Comisionado de las Naciones Unidas para los Refugiados (ACNUR), Fundación Universitaria Claretiana, Diócesis de Quibdó. 2006. Memorias del Desplazamiento Forzado Interno en el Departamento del Chocó. Quibdó.

Ayala-Carcedo, F. 1993. «Estrategias para la reducción de desastres naturales». Investigación y Ciencia 200: 6-13.

Consejo Nacional de Política Económica y Social - Conpes 3470 de 2007. Importancia estratégica del plan de inversiones para garantizar la prestación de los servicios de acueducto, alcantarillado y aseo en el municipio de Quibdó. Bogotá: Ministerio de Medio Ambiente, Vivienda y Desarrollo Territorial. Disponible en: www.dnp.gov.co/archivos/documentos/Subdireccion_Conpes/3470.pdf

Consejo Nacional de Política Económica y Social. CONPES 3146 de 2001. Estrategia para consolidar la ejecución del Plan Nacional para la Prevención y Atención de Desastres (PNAPD) en el corto y mediano plazo. Bogotá: CONPES. Disponible en: www.dnp.gov.co/archivos/documentos/Subdireccion_Conpes/3146.pdf

CORPOSSO - La Red. 2007. Guía Metodológica de DesInventar. Dispnible en: $\mathrm{http} / /$ www.desinventar.org

Departamento Administrativo Nacional (DANE). 2006. Censo del territorio colombiano 2005. Bogotá: DANE.

Departamento Nacional Departamento Administrativo de Salud y Bienestar Social del Chocó (DASALUD), 2004. Estadísticas Sisbén. Quibdó. Quibdó: DASALUD.

Decenio Internacional para la Reducción de los Desastres Naturales (DIRDN). 1996. Día Internacional para la reducción de los desastres. 09 de octubre de 1991. Bogotá: DIRDN

Dirección Nacional de Atención y Prevención de Desastres (DNPAD). 2008. Registro histórico de desastres en el territorio colombiano. Bogotá: DNPAD.

Instituto Colombiano de Geología y Minería (INGEOMINAS) 1999. Mapa de amenaza sísmica en Colombia. Mapa de zonas activas de Colombia. Bogotá: INGEOMINAS

Instituto Geográfico Agustín Codazzi (IGAC). 2006. Chocó características geográficas. Bogotá: IGAC.

Instituto Geográfico Agustín Codazzi (IGAC). Instituto Colombiano de Geología y Minería (INGEOMINAS). 2006. Investigación Integral del Andén Pacífico Colombiano. Bogotá: IGAG-INGEOMINAS.

Lavell, A. 1996. Degradación ambiental, riesgo y desastre urbano. Problemas y conceptos: Hacia la definición de una agenda de investigación. Bogotá: La Red, Tercer Mundo Editores.

Servicio de Información Aeronáutica (AIS). Instituto Colombiano de Geología y Minería (INGEOMINAS). 1997. Estudio general de amenaza sísmica de Colombia, Bogotá. Bogotá: AIS-INGEOMINAS.

Sistema Nacional de para la Atención y Prevención de Desastres. 2008. Datos de Emergencias 1998-2008. Disponible en: www.sigpad.gov.co 